\title{
EFFECT OF ISCHEMIC PRETREATMENT ON HEAT SHOCK PROTEIN 72, NEUROLOGIC OUTCOME, AND HISTOPATHOLOGIC OUTCOME IN A RABBIT MODEL OF SPINAL CORD ISCHEMIA
}

Peter de Haan, MD, $\mathrm{PhD}^{\mathrm{a}}$

Ivo Vanicky, DVM ${ }^{\text {b }}$

Michael J. H. M. Jacobs, MD, $\mathrm{PhD}^{\mathrm{c}}$

Onno Bakker, $\mathrm{PhD}^{\mathrm{d}}$

Jeroen Lips, MS ${ }^{\mathrm{a}}$

Sven A. G. Meylaerts, MD

Cor J. Kalkman, MD, $\mathrm{PhD}^{\mathrm{a}}$
Objective: In the present study, we investigated the effect of ischemic pretreatment on heat shock protein 72 concentration and neurologic and histopathologic outcome after transient spinal cord ischemia.

Methods: In 28 New Zealand White rabbits, an aortic occlusion device was placed infrarenally. The animals were randomly assigned to 2 groups: ischemic pretreatment ( $\mathrm{n}=14$ animals) and control $(\mathrm{n}=14$ animals). The duration of ischemic pretreatment was 6 minutes. After 24 hours, the aorta was occluded for 26 minutes in both groups of animals. Neurologic function was assessed 24 and 48 hours after the definite ischemic insult. At 48 hours, the animals were killed for histopathologic evaluation of the spinal cord. In a separate set of animals, heat shock protein 72 levels were determined in the lumbar spinal cord after both a 6- and 10-minute ischemic period, with the use of a Western blot analysis.

Results: No significant difference in neurologic outcome between the groups was observed at 24 and 48 hours. The incidence of paraplegia and severe paresis at 48 hours was $79 \%$ in the control group and $92 \%$ in the ischemic pretreatment group. There was no difference in histopathologic scores between the groups. Heat shock protein 72 could be clearly detected 1 and 2 days after 6- or 10-minute periods of spinal cord ischemia.

Conclusions: In the present rabbit study, ischemic pretreatment could not induce tolerance against a moderately severe spinal cord ischemic insult, despite increased heat shock protein 72 levels after the preconditioning stimulus. (J Thorac Cardiovasc Surg 2000;120:513-9)
$P$ araplegia is a major complication after operations for thoracoabdominal aortic aneurysm (TAAA). The overall incidence of lower limb neurologic deficits

From the Departments of Anesthesiology, ${ }^{a}$ Vascular Surgery, ${ }^{\mathrm{c}}$ and Endocrinology, ${ }^{\mathrm{d}}$ Academic Hospital, University of Amsterdam, Academic Medical Center, Amsterdam, The Netherlands, and the

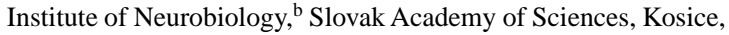
Slovakia. The work was performed at the Department of Experimental and Vascular Surgery, Academic Medical Center, Amsterdam, The Netherlands.

This study was supported by the Dutch Heart Association (grant 97.193).

Received for publication Nov 10, 1999; revisions requested Feb 2, 2000; revisions received Feb 21, 2000; accepted for publication Feb 28, 2000.

Address for reprints: P. de Haan, MD, Department of Anesthesiology, Academic Hospital, University of Amsterdam, Postbus 22660, 1100 DD Amsterdam, The Netherlands (E-mail: P.deHaan@amc.uva.nl).

Copyright (C) 2000 by The American Association for Thoracic Surgery

0022-5223/2000 $\$ 12.00+0 \quad \mathbf{1 2 / \mathbf { 1 } / \mathbf { 1 0 6 8 3 6 }}$

doi:10.1067/mtc.2000.106836 after TAAA surgery was $16 \%$ in a large series of patients. ${ }^{1}$ This complication is the result of a temporary or definitive interruption of spinal cord blood supply. Despite numerous spinal cord protective strategies, a lower extremity neurologic deficit after TAAA surgery remains a distinct possibility. ${ }^{2-6}$

Preconditioning the brain with a nonlethal period of ischemia (insufficient to produce irreversible neuronal damage) has consistently been shown to induce tolerance to a subsequent otherwise lethal ischemic insult 1 to 7 days after the ischemic pretreatment. ${ }^{7,8}$ Although the exact mechanism of the neuroprotective effect of ischemic pretreatment is not fully understood, the acquisition of ischemic tolerance has been related to a reprogramming of gene expression. ${ }^{9,10}$ In particular, the induction of heat shock protein (HSP) 72 is thought to be involved. ${ }^{8}$ It would be advantageous if spinal cord neurons also possessed an inducible endogenous mechanism that increases ischemic tolerance similar to that observed in cerebral neuronal tissue. Ideally, this toler- 


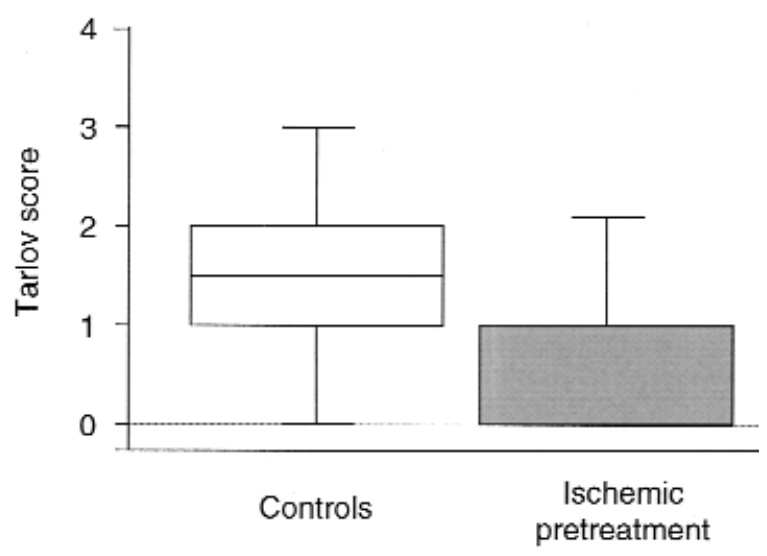

Fig 1. Boxplot of the Tarlov scores of each group of animals at 48 hours. The Tarlov score is on a 5-point scale: 0 (complete paraplegia with no lower-extremity motor function) to 4 (normal motor function). Horizontal bars represent the 90th, 75th, 50th (median), 25th, and 10th percentiles.

ance could then be activated pharmacologically before an anticipated ischemic event. In other models of ischemia, pharmacologic induction of ischemic tolerance has been described. For example, in vitro chemical preconditioning enhanced functional recovery in hippocampal slices after ischemia, ${ }^{11}$ and in a model of myocardial ischemia, the antibiotic herbimycin-A could induce the genetic expression of HSPs and ischemic tolerance. ${ }^{12}$ One recent experiment demonstrated that ischemic pretreatment protected the spinal cord against a mild ischemic insult. ${ }^{13}$ When ischemic preconditioning would induce tolerance against a relatively severe ischemic spinal cord insult (ie, likely to result in paraplegia or severe paresis), the induction of tolerance indeed might allow a new therapeutic approach to minimize the neuronal injury that results from transient spinal cord ischemia during operations for TAAA.

The present study was designed to assess the effect of ischemic pretreatment on neurologic and histopathologic outcome in a rabbit model of moderately severe transient spinal cord ischemia. In addition, spinal HSP 72 levels were determined after sublethal "preconditioning" ischemic periods.

\section{Methods}

Animal care and all procedures were performed in compliance with The National Guidelines for Care of Laboratory Animals in The Netherlands. The study protocol was approved by the Animal Research Committee of the Academic Hospital at the University of Amsterdam, The Netherlands. Twenty-eight New Zealand White rabbits, weighing 3.0 to $3.5 \mathrm{~kg}$, were randomly assigned to 1 of 2 groups: group I, transient spinal cord ischemia (26 minutes) without ischemic pretreatment ( $\mathrm{n}=14$ control animals); group II, transient spinal cord ischemia 24 hours after 6 minutes of ischemic pretreatment ( $\mathrm{n}=14$ animals). HSP 72 production was assessed in the lumbar spinal cord after sublethal "preconditioning" periods of ischemia in an additional group of animals ( $n=8$ animals). To accustom the rabbits to the awake part of the experiment, we domesticated all animals by taking them out of their cages and placing them on the operating table twice daily, starting 7 days before the experiment.

Operative procedure. After being premedicated with ketamine $25 \mathrm{mg} / \mathrm{kg}$ and xylazine $5 \mathrm{mg} / \mathrm{kg}$ intramuscularly, the animals were anesthetized with $1.0 \%$ halothane in a mixture of $50 \%$ oxygen in nitrous oxide by mask. An intravenous catheter (20-gauge) was placed in an ear vein. Normal saline solution was infused at a rate of $4 \mathrm{~mL} / \mathrm{kg}$ per hour. The animals were allowed to breathe spontaneously. Intravenous cephalothin (25 $\mathrm{mg} / \mathrm{kg}$ ) was administered before the incision was made. During the procedure, the heart rate was recorded from the electrocardiograph, and the blood pressure was recorded continuously with a noninvasive blood pressure monitor (Finapress; DatexOhmeda Division, Instrumentarium Corp, Helsinki, Finland). Fur was clipped from the left flank. After preparation of the skin with iodine and infiltration with bupivacaine $0.5 \%(1 \mathrm{~mL})$, a horizontal flank incision was made, and the infrarenal aorta was exposed through a retroperitoneal approach. An aortic occlusion device (Uno, Zevenaar, The Netherlands), consisting of a $2 \mathrm{~F}$ polyethylene snare in a $10.5 \mathrm{~F}$ dual-lumen catheter, was carefully placed around the aorta immediately distal to the renal arteries. The catheter and the snare were tunneled into a subcutaneous pouch on the anterolateral abdomen. The wound was closed in layers, and anesthesia was discontinued.

Ischemic pretreatment. Twenty-four hours after the placement of the snare, the rabbits of groups I and II were placed on the operating table (nonanesthetized), and the noninvasive continuous blood pressure monitor was placed around the left hind leg. The wound was infiltrated with bupivacaine $0.25 \%$ ( $2 \mathrm{~mL}$ ). One or 2 stitches of the operative wound were opened, and the snare tubing was retrieved. In group II, the aorta was occluded for 6 minutes by a tightening of the snare through the dual-lumen catheter. Definitive occlusion was confirmed by the loss of pulsatile pressure on the continuous blood pressure monitor. Reperfusion was verified by the reappearance of the pulsatile pressure waveform. Thereafter, the wound was closed, and all animals were placed back in their cages.

Spinal cord ischemia. Twenty-four hours after ischemic pretreatment, the animals of groups I and II were placed on the operating table, and the continuous blood pressure monitor was placed around the left hind leg. The wound was infiltrated with $2 \mathrm{~mL}$ of $0.25 \%$ bupivacaine, and the same stitches were reopened to retrieve the snare tubing. The aorta was occluded for 26 minutes in all animals while they were awake. Rectal temperature was recorded continuously before, during, and after the ischemic period. Pressure distal to the occlusion device was recorded continuously before, during, 
and for 15 minutes after the occlusion period. Aortic occlusion and reperfusion were confirmed by the loss and reappearance of pulsatile blood pressure.

Neurologic scoring system. The animals were neurologically scored on a 5-point scale (modified Tarlov score) 24 hours after the ischemic pretreatment and 24 and 48 hours after the definite ischemic insult by a blinded observer: 0 , paraplegia with no lower-extremity motor function; 1, poor lower-extremity motor function (flicker of movement or weak antigravity movement only); 2, some lower-extremity motor function with good antigravity strength but an inability to draw legs under body and/or hop; 3 , the ability to draw legs under body and hop but not normally; 4, normal motor function. Depending on the severity of the neurologic dysfunction, bladder contents were expressed twice daily with the Credé maneuver.

Spinal cord histopathologic features. Two days after the definite ischemic insult, the animals were anesthetized with $1 \%$ halothane by mask after premedication with ketamine 25 $\mathrm{mg} / \mathrm{kg}$ and xylazine $5 \mathrm{mg} / \mathrm{kg}$ intramuscularly. The animals were given $2500 \mathrm{U}$ of heparin and were killed with $100 \mathrm{mg}$ of pentobarbital. Subsequently, the animals were perfusion fixated with $3.6 \%$ formalin after being flushed with normal saline solution. The lumbosacral portion of the spinal cord was taken out en bloc, and the spinal cord was carefully removed. Formalin was used as a fixative for at least 10 days.

The L2 to L5 segments were dissected in 12 pieces. A 1$\mathrm{mm}$ block of tissue was taken from each piece. The blocks were embedded in paraffin, and 4-mm thick sections were stained with hematoxylin and eosin. One section from each block was evaluated by an experienced histopathologist (I.V.) who was unaware of the experimental conditions. Histopathologic changes of the gray matter were scored on a 7-point scale: 0 , no lesion observed; 1 , gray matter contained 1 to 5 eosinophilic neurons; 2 , gray matter contained 5 to 10 eosinophilic neurons; 3 , gray matter contained more than 10 eosinophilic neurons; 4 , small infarction (less than one third of the gray matter area); 5 , moderate infarction (one third to one half of the gray matter area); and 6, large infarction (more than one half of the gray matter area). The scores from all the sections from each spinal cord were averaged to give a final score for an individual animal.

Western blot analysis of spinal cord HSP 72 content. The production of HSP 72 in the lower lumbar portion of the spinal cord was assessed after sublethal "preconditioning" ischemic periods in 7 animals. The snare to produce spinal cord ischemia was applied as described earlier. After 24 hours, the animals were placed on the operating table (in the awake state), and the aorta was occluded. Aortic occlusion and reperfusion was confirmed by the loss and reappearance of pulsatile blood pressure with the noninvasive continuous blood pressure monitor. In 5 animals, the aorta was occluded for 6 minutes. HSP 72 content was determined after 24 hours $(n=$ 3 animals) and after 48 hours ( $n=2$ animals). In 2 animals, the aorta was occluded for 10 minutes, and the HSP 72 content was determined after 48 hours. So that spinal cord tissue could be obtained at the L4 to L5 level, the animals were killed with

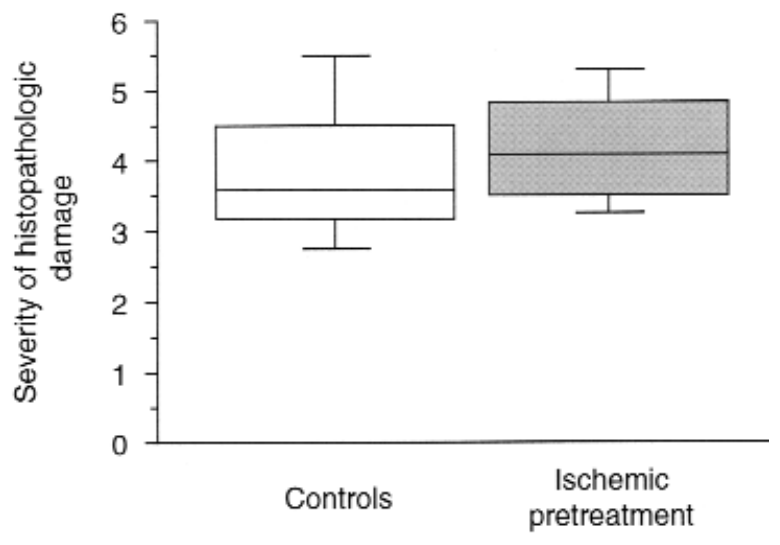

Fig 2. Boxplot of the histopathologic scores of each group of animals. Histopathologic score is on a 7-point scale: 0 (normal) to 6 (severe gray matter necrosis). Horizontal bars represent the 90th, 75th, 50th (median), 25th, and 10th percentiles.

$300 \mathrm{mg}$ sodium pentobarbital (Nembutal), and the cord was quickly harvested with the plunger of a $1-\mathrm{mL}$ syringe and immediately frozen in liquid nitrogen. HSP 72 content was semiquantitatively determined with a Western blot analysis, with chemoluminescence. One normal spinal cord was used to measure baseline chemoluminescence. The spinal cord samples were lysed with an UltraTurrax homogenizer (Jahnke und Kinkel, Staufen, Germany). The lysate was centrifuged $(15,000 \mathrm{~g}$ for 10 minutes), and the supernatant was frozen $\left(-70^{\circ} \mathrm{C}\right)$. Lysate $(10 \mu \mathrm{L})$ was mixed with $10 \mu \mathrm{L}$ loading buffer (protein concentration, $60 \mathrm{mg} / \mathrm{mL}$ ) and was applied to a standard $10 \%$ sodium dodecylsulfate-polyacrilamide gel electrophoresis and electroblotted onto a polyvinylidenedifluoride membrane. The blot was blocked with $5 \%$ casein-hydrolysate (Boehringer Mannheim, Germany) in phosphate-buffered saline solution (PBS). The first antibody (anti-HSP 72, MA 3007; Affinity BioReagents, Inc, Golden, Colo) was diluted $1: 1000$ in $5 \%$ casein/PBS and incubated for 1 hour at $30^{\circ} \mathrm{C}$. The gel was washed $(3 \times 10$ minutes) with $\mathrm{PBS} / 0.05 \% \mathrm{NP} 40$. Thereafter the second antibody (goat-anti mouse peroxidaseconjugated, Boehringer Mannheim) was administered (diluted $1: 1000$ in $5 \%$ casein/PBS) and incubated for 1 hour at $30^{\circ} \mathrm{C}$. The gel was washed $(3 \times 10$ minutes $)$, and the chemolumiscent peroxidase substrate (LumiLight ${ }^{\text {plus; }}$ Boehringer Mannheim) was added for 5 minutes. Thereafter, quantification and detection was performed with the Lumi-Imager device (Boehringer Mannheim). Recombinant pure HSP 72 (StressGen, Biotechnologies Corp, Vancouver, British Columbia, Canada) was used as positive control (200 ng).

Statistical analysis. The duration of aortic occlusion was chosen to produce $80 \%$ paraplegia and severe paresis (Tarlov score, $0,1,2)$ in the control group. Awake aortic occlusion of 26 minutes was observed in several studies to produce the required rate and severity of neurologic deficits. ${ }^{14,15} \mathrm{~A}$ sample size of 14 animals in each group was necessary to acquire suf- 
ficient power $(1-\beta, 0.80 ; \alpha, 0.05)$ to demonstrate a decrease in the incidence of paraplegia and severe paresis from 0.8 in control animals to 0.3 in the preconditioning group.

Physiologic data are expressed as means \pm SD. Blood pressures distal to the occlusion device were averaged before, during, and 15 minutes after aortic occlusion. Neurologic outcome and histopathologic scores are expressed as medians and 10th to 90th percentiles. The Mann-Whitney $U$ test was used to compare the histopathologic score, the neurologic score (Tarlov), and physiologic data. The Fisher exact test was used for the comparison of the overall incidence of neurologic deficits, the incidence of paraplegia, the incidence of paraparesis, and the incidence of paraplegia and severe paraparesis.

\section{Results}

Distal pressures before aortic occlusion were $88 \pm 14$ $\mathrm{mm} \mathrm{Hg}$ and $84 \pm 9 \mathrm{~mm} \mathrm{Hg}$ in the control and ischemic pretreatment groups, respectively $(P=.49)$. During occlusion, pressures were $8 \pm 4 \mathrm{~mm} \mathrm{Hg}$ and $10 \pm 3 \mathrm{~mm}$ $\mathrm{Hg}(P=.08)$. After the occlusion, blood pressures returned to $85 \pm 14 \mathrm{~mm} \mathrm{Hg}$ and $77 \pm 15 \mathrm{~mm} \mathrm{Hg}$, respectively $(P=.15)$. Temperatures were $39.0^{\circ} \mathrm{C} \pm 0.5^{\circ} \mathrm{C}$ and $39.3^{\circ} \mathrm{C} \pm 0.5^{\circ} \mathrm{C}$ at the start of aortic occlusion in the control and ischemic pretreatment groups, respectively $(P=.22)$. During occlusion, temperatures slightly decreased to $38.8^{\circ} \mathrm{C} \pm 0.5^{\circ} \mathrm{C}$ in the control group and $38.9^{\circ} \mathrm{C} \pm 0.5^{\circ} \mathrm{C}$ in the ischemic pretreatment group $(P=.57)$. After 15 minutes of reperfusion, temperatures were $38.9^{\circ} \mathrm{C} \pm 0.4^{\circ} \mathrm{C}$ in the control group and $38.9^{\circ} \mathrm{C} \pm$ $0.4^{\circ} \mathrm{C}$ in the ischemic pretreatment group $(P=.87)$.

Neurologic outcome. The Tarlov scores are provided as a boxplot in Fig 1. Overall neurologic outcomes are shown in Table I. After 24 and 48 hours, no significant difference in neurologic outcome between the groups was observed. After 24 hours, the Tarlov scores were 1 (0-3) and 0.5 (0-2.1); after 48 hours, the scores were $1.5(0-3)$ and $1(0-2.1)$ in the control and ischemic pretreatment groups, respectively. Eleven of 14 animals (79\%) in the control group and 13 of 14 animals (92\%) in the pretreatment group had paraplegia or severe paresis at 48 hours. Ten animals in both groups required expression of bladder contents.

Histopathologic scores. Histopathologic scores in the control and ischemic pretreatment groups were 3.6 (2.8-5.5) and 4.1 (3.2-5.3), respectively $(P=.31$; Fig 2). In general, either minimal damage involving a few necrotic neurons or infarctions were detected. In most animals, the infarctions were found in the lower lumbar segments only, but in some animals the whole lumbar spinal cord was severely damaged. Selective neuronal necrosis was rarely seen, but in 1 animal numerous eosinophilic neurons were found in all segments. In 3 animals ( 2 control and 1 ischemic pretreatment), the spinal cord was not adequately perfusion fixated, and the spinal cords could not be evaluated.

HSP 72. Neurologic deficits were not observed in the animals in which HSP 72 was determined after 6 or 10 minutes of spinal cord ischemia. The chemoluminescent data demonstrated that the HSP 72 content was elevated in the lower lumbar spinal cords of all 7 animals that were subjected to sublethal durations of spinal cord ischemia (Fig 3). The relative HSP 72 increase, as compared with the nonischemic control spinal cord sample, was highest in the 3 animals that were killed 24 hours after 6 minutes of spinal cord ischemia $(370 \%, 240 \%$, and $230 \%$ ), whereas the relative HSP 72 increase 48 hours after 6 minutes of spinal cord ischemia was $180 \%$ and $160 \%$. In the 2 animals that were subjected to 10 minutes of spinal cord ischemia, the relative HSP 72 increase after 48 hours was $170 \%$ and $160 \%$ (Fig 4).

\section{Discussion}

In the present rabbit study, 6 minutes of ischemic pretreatment 24 hours before a moderately severe spinal cord ischemic insult could not improve neurologic and histopathologic outcome. In a separate set of animals, a similar ischemic period (as that used for the preconditioning) clearly increased HSP 72 concentration.

Infrarenal aortic occlusion in the rabbit is a reproducible model to produce transient spinal cord ischemia. The duration of ischemia correlates with histopathologic injury and with the degree of functional impairment. ${ }^{16}$ In the present study, the placement of an aortic occlusion device permitted repeated awake occlusions of the aorta, which we could confirm with a noninvasive continuous blood pressure monitor.

The acquisition of ischemic tolerance by ischemic pretreatment has been related to the induction of HSPs. The HSPs prevent the disruption of proteins and bind to abnormal proteins until they are refolded or disintegrated. The expression pattern of HSP, especially the $72-\mathrm{kDa}$ HSP, in ischemic preconditioning was described to be compatible with the dose response and time window of ischemic tolerance. ${ }^{17}$ In the present study, HSP 72 was measured to validate the experimental model (ie, to assess whether the 6 minutes of ischemic pretreatment was sufficiently severe to result in HSP 72 production). HSP 72 was determined after 2 durations of ischemic pretreatment and 2 time windows between pretreatment and definite insult to confirm that the global pattern of HSP 72 expression was in agreement with the present literature. 
Table I. Neurologic outcomes after temporary spinal cord ischemia (26 minutes) in the control ( $n=14$ animals) and ischemic pretreatment $(n=14$ animals) group at 48 hours

\begin{tabular}{lrrrr}
\hline & \multicolumn{2}{c}{ Control } & Ischemic pretreatment & P value \\
\hline Tarlov score* (range) $^{2}$ & $1.5(0-3)$ & 1 (0-2.1) & .12 \\
Overall incidence of neurologic deficits $^{\dagger}(\%)$ & $14 / 14(100)$ & $14 / 14(100)$ & 7.99 \\
Incidence of complete paraplegia $^{\ddagger}(\%)$ & $3 / 14(21)$ & $6 / 14(43)$ & .42 \\
Incidence of paraparesis $^{(\%)}$ & $11 / 14(79)$ & $8 / 14(57)$ & .42 \\
Incidence of paraplegia and severe paraparesis $(\%)^{11 / 14}(79)$ & $13 / 14(92)$ & .60 \\
\hline
\end{tabular}

*The Tarlov score is on a 5-point scale: 0 (complete paraplegia; no lower-extremity motor function) to 4 (normal motor function).

†Tarlov 0-3.

†Tarlov 0.

\$Tarlov 1-3.

| Tarlov 0, 1, and 2.

The protection provided by ischemic preconditioning is dependent on the duration of ischemic pretreatment, the time window between pretreatment and subsequent insult, and the severity of the subsequent ischemic insult. These variables allow numerous combinations. Therefore, the lack of neuroprotection by ischemic preconditioning in the present study might be explained both by a too long or too short duration of ischemic pretreatment. Indeed, the duration of the preconditioning stimulus might have been too long, as suggested by the increased incidence of severe neurologic deficits in the ischemic pretreatment group (92\% vs $79 \%$ ) at 48 hours. However, this is unlikely because a relatively long period of ischemic pretreatment (10 minutes) was found to be neuroprotective in a similar model of mild spinal cord ischemia (15-minute duration). ${ }^{13}$ These longer periods of ischemic pretreatment carry the risk of producing irreversible damage by themselves. Drummond and Moore ${ }^{18}$ described that the awake duration of spinal cord ischemia that had a $50 \%$ chance to produce neurologic deficits was approximately 12 minutes in unanesthetized rabbits. By way of avoiding the possible induction of irreversible damage, the duration of ischemic pretreatment should be considerably shorter. The duration of ischemic pretreatment in the present study (6 minutes) might have been too short to induce tolerance. However, this seems improbable because the acquisition of ischemic tolerance with ischemic preconditioning is thought to be mediated by HSP $72 .{ }^{17}$ In the present experiment, HSP 72 was clearly increased after 2 sublethal ischemic periods, with HSP 72 content being maximal 24 hours after a 6minute period of spinal cord ischemia. In addition, a recent rabbit study showed that 5 minutes of ischemic preconditioning could enhance the tolerance of the spinal cord to ischemia. ${ }^{19}$

For several reasons, we opted to investigate the effect of ischemic pretreatment after an interval of 24 hours.
HSP 72, which is thought to be important for the acquisition of neuroprotection with ischemic preconditioning, was mainly produced in the rabbit spinal cord between 8 and 24 hours after the ischemic pretreatment, whereas HSP 72 levels were lower at 48 hours. ${ }^{13}$ This trend of expression was confirmed in the present study. In models of cerebral ischemia, a 24-hour interval between ischemic pretreatment and the subsequent ischemic insult resulted in ischemic tolerance. ${ }^{7,8,20} \mathrm{In}$ addition, occlusion of several segmental arteries in the rabbit resulted in tolerance to a spinal cord ischemic insult within a 24-hour interval. ${ }^{21}$ The protection achieved with this method was attributed to ischemic preconditioning.

In a model of temporary spinal cord ischemia in the Japanese White rabbit, 15 minutes of ischemia 2 days after 10 minutes of ischemic pretreatment improved functional and histopathologic outcome. ${ }^{13}$ In that study, the ischemic insult produced only mild neurologic deficits in the control group (Tarlov scores 0 and 1 were not observed). Therefore, the most likely explanation for the lack of protection by ischemic pretreatment in the present study is that the duration of the second ischemic insult was too long. However, our study was designed to evaluate whether ischemic pretreatment might protect against a clinically relevant (moderately severe) ischemic insult, which would result in a substantial rate of paraplegia or severe paraparesis. During TAAA surgery, the induction of ischemic tolerance by pharmacologic agents or other means can be clinically valuable if tolerance could be induced against a moderately severe ischemic insult. In this respect, it is important to point out that, after TAAA surgery, a mild paraparesis is likely to resolve and that most of these patients are able to regain their preoperative level of functioning. ${ }^{1}$ Instead, paraplegia or severe paraparesis is the most challenging problem, because severe deficits result in permanent disablement and are 


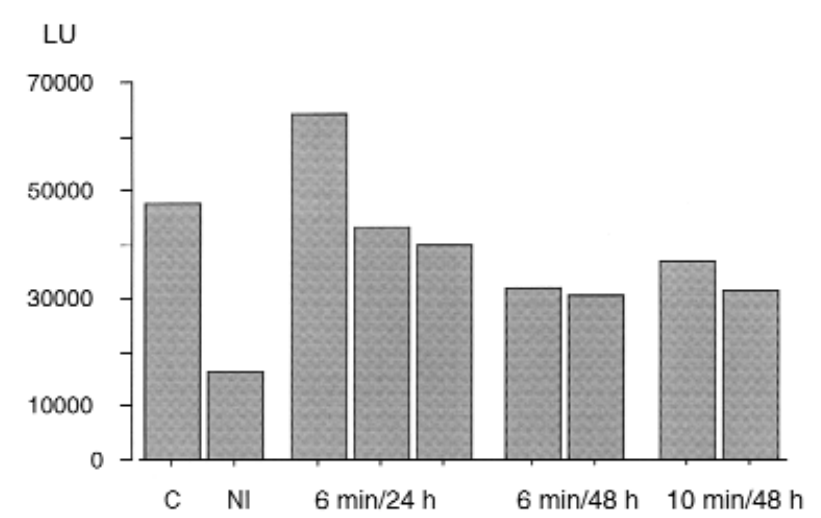

Fig 3. HSP 72 content in each sample depicted as the raw chemoluminescent data from the Lumi-Imager device. The vertical axis represents chemoluminescence in light units (LUs). C, Positive control (reference sample containing 200ng pure HSP 72); NI, spinal cord of the nonischemic animal; $6 \mathrm{~min} / 24 h, 24$ hours after 6 minutes of spinal cord ischemia; $6 \mathrm{~min} / 48 \mathrm{~h}, 48$ hours after 6 minutes of spinal cord ischemia; $10 \mathrm{~min} / 48 \mathrm{~h}, 48$ hours after 10 minutes of spinal cord ischemia.

associated with both a high incidence of comorbidity and an increased mortality outcome. ${ }^{1,4}$ In the present study, $79 \%$ of the control animals had paraplegia or severe paraparesis. Therefore, our results suggest that the ability to achieve relevant spinal cord protection with ischemic pretreatment is limited.

In conclusion, in a rabbit model of temporary spinal cord ischemia, ischemic pretreatment could not induce tolerance against a moderately severe ischemic insult, although the preconditioning stimulus clearly increased HSP 72 levels.

We thank Marloes Klein, Godelieve Huyzer, and Sjohn Dries for biotechnical assistance.

\section{REFERENCES}

1. Svensson LG, Crawford ES, Hess KR, Coselli JS, Safi HJ. Experience with 1509 patients undergoing thoracoabdominal aortic operations. J Vasc Surg 1993;17:357-68.

2. Crawford ES, Mizrahi EM, Hess KR, Coselli JS, Safi HJ, Patel VM. The impact of distal aortic perfusion and somatosensory evoked potential monitoring on prevention of paraplegia after aortic aneurysm operation. J Thorac Cardiovasc Surg 1988;95: 357-67.

3. Kouchoukos NT, Daily BB, Rokkas CK, Murphy SF, Bauer S, Abboud N. Hypothermic bypass and circulatory arrest for operations on the descending thoracic and thoracoabdominal aorta. Ann Thorac Surg 1995;60:67-76.

4. de Haan P, Kalkman CJ, de Mol BA, Ubags LH, Veldman DJ, Jacobs MJ. Efficacy of transcranial motor-evoked myogenic

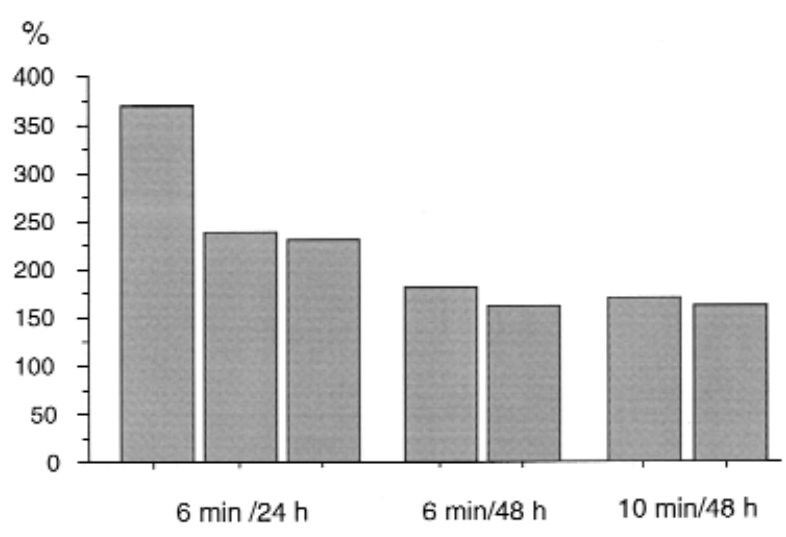

Fig 4. HSP 72 content as relative light units per milligram of protein (expressed as percentage of baseline from the nonischemic spinal cord sample). $6 \mathrm{~min} / 24 h, 24$ hours after 6 minutes of spinal cord ischemia; $6 \mathrm{~min} / 48 \mathrm{~h}, 48$ hours after 6 minutes of spinal cord ischemia; $10 \mathrm{~min} / 48 \mathrm{~h}, 48$ hours after 10 minutes of spinal cord ischemia.

potentials to detect spinal cord ischemia during operations for thoracoabdominal aneurysms. J Thorac Cardiovasc Surg 1997;113:87-100.

5. Safi HJ, Miller CC, Carr C, Iliopoulos DC, Dorsay DA, Baldwin JC. Importance of intercostal artery reattachment during thoracoabdominal aortic aneurysm repair. J Vasc Surg 1998;27:58-66.

6. Svensson LG, Hess KR, D'Agostino RS, Entrup MH, Hreib K, Kimmel WA, et al. Reduction of neurologic injury after high-risk thoracoabdominal aortic operation. Ann Thorac Surg 1998;66: 132-8.

7. Kato H, Liu Y, Araki T, Kogure K. Temporal profile of the effects of pretreatment with brief cerebral ischemia on the neuronal damage following secondary ischemic insult in the gerbil: cumulative damage and protective effects. Brain Res 1991;553:238-42.

8. Kirino T, Tsujita Y, Tamura A. Induced tolerance to ischemia in gerbil hippocampal neurons. J Cereb Blood Flow Metab 1991;11:299-307.

9. Sommer C, Gass P, Kiessling M. Selective c-JUN expression in CA1 neurons of the gerbil hippocampus during and after acquisition of an ischemia-tolerant state. Brain Pathol 1995;5:135-44.

10. Belayev L, Ginsberg MD, Alonso OF, Singer JT, Zhao W, Busto $\mathrm{R}$. Bilateral ischemic tolerance of rat hippocampus induced by prior unilateral transient focal ischemia: relationship to c-fos mRNA expression. Neuroreport 1996;8:55-9.

11. Riepe MW, Esclaire F, Kasischke K, Schreiber S, Nakase H, Kempski O, et al. Increased hypoxic tolerance by chemical inhibition of oxidative phosphorylation: "chemical preconditioning." J Cereb Blood Flow Metab 1997;17:257-64.

12. Morris SD, Cumming DV, Latchman DS, Yellon DM. Specific induction of the 70-kD heat stress proteins by the tyrosine kinase inhibitor herbimycin-A protects rat neonatal cardiomyocytes: A new pharmacological route to stress protein expression? J Clin Invest 1996;97:706-12.

13. Sakurai M, Hayashi T, Abe K, Aoki M, Sadahiro M, Tabayashi K. Enhancement of heat shock protein expression after transient ischemia in the preconditioned spinal cord of rabbits. J Vasc Surg 1998;27:720-5. 
14. Madden KP, Clark WM, Marcoux FW, Probert AJ, Weber ML, Rivier J, et al. Treatment with conotoxin, an "N-type" calcium channel blocker, in neuronal hypoxic-ischemic injury. Brain Res 1990;537:256-62.

15. Madden KP, Clark WM, Zivin JA. Delayed therapy of experimental ischemia with competitive N-methyl-d-aspartate antagonists in rabbits. Stroke 1993;24:1068-71.

16. Zivin JA, DeGirolami U. Spinal cord infarction: a highly reproducible stroke model. Stroke 1980;11:200-2.

17. Chen J, Simon R. Ischemic tolerance in the brain. Neurology 1997;48:306-11.

18. Drummond JC, Moore SS. The influence of dextrose administra- tion on neurologic outcome after temporary spinal cord ischemia in the rabbit. Anesthesiology 1989;70:64-70.

19. Fan T, Wang CC, Wang FM, Cheng F, Qiao H, Liu SL, et al. Experimental study of the protection of ischemic preconditioning to spinal cord ischemia. Surg Neurol 1999;52:299305.

20. Kitagawa K, Matsumoto M, Tagaya M, Hata R, Ueda H, Niinobe $\mathrm{M}$, et al. "Ischemic tolerance" phenomenon found in the brain. Brain Res 1990;528:21-4.

21. Vacanti FX, Kwun BD. Vascular occlusion produced over 24 hours increases spinal cord tolerance to occlusion. J Surg Res 1996;62:29-31. 